\title{
La investigación-acción cooperativa como vía de formación y transformación curricular: Una experiencia práctica hacia la educación intercultural
}

\author{
Cooperative research action as a means of training and curriculum change: \\ A practical experience to intercultural education

\begin{abstract}
A pesquisa-ação cooperativa como vía de formação e transformação curricular: Uma experiência prática para a educação intercultural
\end{abstract}

\author{
González Mediel, Olga ${ }^{a}$; Berríos Valenzuela, Llarela ${ }^{b}$; Vila-Fagundes, Caterine ${ }^{c}$ \\ aDepartamento de Didáctica de la Expresión Musical y Corporal, \\ Universitat de Barcelona, Barcelona, España. \\ Teléfono: 34-934035134. Correo electrónico: ogonzalez@ub.edu \\ bInstituto Ciencias de la Familia, Universidad de los Andes, Las Condes, Santiago, Chile. \\ Teléfono: 56- 26181601. Correo electrónico: 1larela.berrios@uandes.cl \\ 'Departamento de Políticas y Gestión de la Educación, \\ Universidad Federal do Rio Grande do Sul, Porto Alegre, Brasil. \\ Teléfono: 51-33083266. Correo electrónico: caterine.fagundes@gmail.com
}

\begin{abstract}
RESUMEN
El artículo presenta una experiencia de investigación-acción cooperativa como vía de formación del profesorado y de transformación curricular. Tiene como objetivo general iniciar una transformación del currículum del ciclo inicial de un Centro de Educación Primaria para avanzar a un modelo efectivo de educación intercultural utilizando, como desencadenante, un recurso musical. Para lograr el objetivo se intervino en un centro educativo público de primaria ubicado en la provincia de Barcelona. Sobre la base de las necesidades del centro escogimos, para nuestro trabajo, un diseño metodológico que nos permitiera plantear una investigación "transformadora" hacia el centro y "formadora" hacia el profesorado participante. Los resultados apuntan a que efectivamente se produjo la transformación curricular basada en la educación intercultural, todo esto asociado al proceso de formación del profesorado, la elaboración de estrategias metodológicas, el currículum y el alumnado.
\end{abstract}

Palabras clave: educación intercultural, investigación-acción, transformación curricular, formación del profesorado.

\begin{abstract}
The paper presents an experience of cooperative action-research through teacher training and curriculum transformation. As the main general objective, there is the intention to begin a transformation in the curriculum of the initial cycle of a Center for Primary Education to move forward to an effective model of intercultural education, using as trigger a musical feature. To achieve the proposed objective an intervention in a public elementary school located in the province of Barcelona was held. Based on the needs of the center chosen to develope this work, a methodological design that allowed to propose a "transformative" research for the center and "instructive" for the participating teachers was applied. The main results suggest curriculum changes with respect to interculturalism, closely associated with the teachers' training, methodological strategies, curriculum and students.
\end{abstract}

Key words: intercultural education, action research, academic transformation, teacher training. 


\section{RESUMO}

Apresenta-se uma experiência de pesquisa-ação cooperativa como via de formação de professores e de transformação curricular. Objetivou-se principalmente, iniciar uma transformação curricular no ciclo inicial de um Centro de Educação Primária para avançar a um modelo efetivo de educação intercultural, empregando um recurso musical como fator desencadeante. Para atingir o objetivo proposto, realizou-se uma intervenção em um centro educativo público de primário localizado na província de Barcelona. Baseando-nos nas necessidades do centro, escolhemos para esse trabalho, um projeto metodológico que nos permitisse propor uma pesquisa "transformadora" para o centro e, "formadora", para os professores participantes. Resultados apontam que efetivamente ocorreram transformações curriculares baseadas na educação intercultural, estreitamente associadas ao processo de formação dos professores, à elaboração de estratégias metodológicas, ao currículo e aos estudantes.

Palavras chave: educação intercultural, pesquisa-ação, transformação curricular, formação de professores.

\section{INTRODUCCIÓN}

La sociedad que nos encontramos es hoy, más que nunca, una sociedad multicultural gracias sobre todo al fenómeno de la globalización y a los efectos de las diferentes migraciones. Los sistemas de valores y los patrones de conducta en uso no dan una respuesta eficiente a esta nueva realidad plural (Delgado, 1998; Goytisolo y Näir, 2000; Näir, 2001; De Lucas, 2003; Palou, 2010).

La escuela, fiel reflejo de la sociedad que la acoge, no puede seguir transmitiendo una única visión del mundo desde su propio prisma cultural. Para dar respuesta a esta nueva realidad aparecen nuevos enfoques educativos entre los cuales destaca el modelo de educación intercultural, el cual promueve el diálogo como puntal para conseguir la creación de nuevos espacios culturales donde todo el mundo tenga cabida y se sienta reconocido y apreciado (Gelpi, 1998; Siguan, 1998; Aja, 2000; Terren, 2003; Alegre, 2005a, 2005b; Jordán, 2007a, 2007b). También destacan investigaciones como las de Bartolomé (1997), Eurydice (2004), Pérez y Rahona (2005), Eresta y Delpino (2007), Etxeberría y Elosegui (2010), Palou (2010), Pavez (2011) y Mussino \& Strozza (2012).

Así como las personas necesitan desarrollar una identidad sólida, los colectivos también necesitan definir sus propias identidades. Para esto, las sociedades necesitan desarrollar el pluralismo cultural. Stickel (1987: 34) expone cuatro condiciones necesarias para que pueda crecer el pluralismo cultural:

- Existencia de diversidad cultural dentro de la sociedad

- Interacción inter e intragrupos

- Los grupos deben compartir aproximadamente las mismas oportunidades políticas, económicas y educativas

- La sociedad debe valorar la identidad cultural de los grupos y de las personas

En cualquiera de estos casos, para el desarrollo del interculturalismo es condición fundamental que los distintos grupos adopten una actitud positiva hacia el reconocimiento de las identidades de los otros para que se puedan desarrollar competencias interculturales. Sobre el desarrollo de competencias interculturales, Perry \& Southwell (2011) exponen que la enseñanza de la competencia intercultural requiere el desarrollo de la conciencia cultural crítico. Desde la misma perspectiva Talkington, Lengel \& Byram (2004) 
argumentan que la enseñanza de la cultura "(por ejemplo, los aspectos institucionales, históricos y políticos de la cultura) no es suficiente, y que el desarrollo de la competencia intercultural requiere la enseñanza de la cultura subjetiva, en el que la atención se centra en la exploración de las visiones del mundo alternativas y la autoconsciencia culturales (Bennett, 2009). Todos estos teóricos sostienen que el conocimiento cultural por sí solo no conduce a la competencia intercultural. Los estudiantes deben examinar críticamente la cultura (no solo acumular hechos y conocimientos sobre la cultura) para que haya desarrollo de la competencia intercultural.

La investigación acción colaborativa demanda una profunda modificación curricular que permita incorporar este enfoque, y para ello se necesitan los elementos que faciliten la puesta en marcha por parte de todos los agentes del centro educativo.

La música como materia educativa dentro del currículum de educación primaria específica, desde el primer nivel de concreción, un elemento de uso directo en el aula con una potente carga cultural: la canción infantil en otras lenguas. Inscrita en este marco, los antecedentes empíricos de una investigación que utiliza como detonante la canción como recurso didáctico para iniciar un proceso de cambio curricular en el ciclo inicial de una escuela de educación primaria.

Para plantear el problema que centra nuestra búsqueda hemos considerado el marco teórico y práctico desarrollado alrededor de la educación intercultural. De acuerdo con las diferentes líneas de investigación sobre la educación intercultural (Banks, 2006; Bartolomé, 1997; Besalú, 2002; Jordán, 1996), estas problemáticas fundamentalmente son de tipo práctico y responden a preocupaciones temáticas susceptibles de un cambio según Bartolomé y Sandín (2001), Palou (2010), Tosolini, Giusti y Papponi Morelli (2007). De todas ellas destacamos las siguientes:

- Los currículums son monoculturales.

- No hay recursos específicos adecuados a la escuela.

- Falta formación específica del profesorado en cuanto a educación intercultural y conocimiento de diferentes realidades culturales.

- Hay más material y directrices de trabajo para secundaria que para primaria.

- La diversidad es cada vez más grande, y los libros de texto introducen cambios muy lentamente mientras la realidad cambia muy rápidamente.

- Estas constataciones y carencias conocidas nos hicieron plantear varias interrogantes que han sido el punto de partida de nuestro trabajo.

\section{¿Cómo conseguir transformar un currículum?}

La necesidad de conseguir una transformación práctica del currículum nos hizo plantear también otras cuestiones:

- ¿Qué tipos de recursos podríamos utilizar?

- ¿Qué tipo de formación para el profesorado podríamos establecer?

El convencimiento de que no se puede establecer un diálogo intercultural si no hay un cierto conocimiento cultural, nos hizo plantear un plan de acción o hipótesis-acción: desde el trabajo con canciones en otras lenguas será más fácil introducir el conocimiento 
de otras culturas en las aulas, como primer paso para su reconocimiento y posterior aprecio. Así pues, la canción popular infantil de diferentes países sería el recurso que, inicialmente, recopilaríamos y usaríamos para utilizarlo como desencadenante de la transformación curricular en todas las áreas.

Utilizar la canción en diferentes lenguas estaría de acuerdo con las orientaciones de Jordán (1996), Navarro (1994) y Besalú (2002) y respeto a la introducción de nuevos contenidos en el currículum para acercarlo a un modelo intercultural. El mismo recurso musical introduciría también el conocimiento de otras lenguas y, en las más próximas, permitiría también el trabajo a base de las semblanzas. Los cuentos serían otros recursos interesantes para conocer otras formas de ver y de ser, susceptibles de ser trabajados en el área de lengua. Los juegos psicomotrices de los diversos orígenes permitirían también incluir nuevos contenidos en educación física. El conocimiento del medio natural y social podría abarcar muchas posibilidades de trabajo, empezando por el mejor conocimiento de las culturas presentes en el aula y teniendo en la visualización de los diferentes países en una proyección del mapa de Peters, aparecido en 1983, la mejor manera de ubicar nuestro país y los otros en el mundo. El trabajo sensorial de proporciones y medidas, unido a los datos conocidos en conocimiento del medio sobre los diferentes países sería una actividad interesante a trabajar en el área de matemáticas, así como el conocimiento de otras formas de contar y medir. Finalmente, el amplio abanico de posibilidades de trabajo desde el área de educación visual y plástica siempre permitiría redondear el trabajo hecho desde la transformación curricular a las otras áreas tomando algún elemento conocido y fijándolo mediante un dibujo.

El profesorado tiene un rol esencial en la integración del alumnado inmigrante (Bartolomé, 2002; Sadker, Sadker \& Zittleman, 2008; Eurydice, 2009; Palou, 2010). Sin embargo, no tienen las herramientas necesarias para poder realizar este proceso de forma adecuada pues se requiere el desarrollo de conocimientos, competencias, actitudes y didáctica específicas (Rubio, 2009; Atwater, 2010; Aranda, 2011; Reid \& Sripraks, 2012). Por tanto, la formación que podríamos aportar al profesorado entendíamos que debería estar vinculada a la práctica, y la metodología de investigación escogida, teníamos que aunar, la teoría y la práctica. El resto de recursos a utilizar para conseguir una transformación en todas las áreas para acercarlas a un modelo educativo intercultural, se debería elaborar desde la reflexión conjunta del equipo investigador y profesorado, ajustando las orientaciones y el material de trabajo existente de cariz intercultural.

La transformación curricular dependería de varios factores. En el tercer nivel de concreción (trabajo en las aulas), podríamos trabajar directamente, modificando el currículum desde el momento que fuéramos cambiando la práctica educativa del profesorado, (elaboración y aplicación de recursos, nuevas estrategias de enseñanza-aprendizaje, etcétera). El currículum, en el segundo nivel de concreción, se haría eco en la medida que la experiencia investigadora fuera positiva.

Estas interrogantes y las hipótesis de acción que se desprenden, nos permitieron detallar el objetivo general y los objetivos específicos del trabajo investigador que finalmente logramos conseguir, y que planteaban el iniciar una transformación del currículum del ciclo inicial de un centro de educación primaria para que avanzase hacia un modelo efectivo de educación intercultural utilizando como desencadenante un recurso musical.

Debido a que un verdadero proceso de transformación curricular ha de afectar diferentes elementos de la comunidad educativa, ajustamos los objetivos específicos del 
trabajo investigador para que incluyeran la formación del propio profesorado (respecto del modelo de educación intercultural, la adopción de nuevas estrategias de aprendizaje, la inclusión de nuevos contenidos culturales, el desarrollo de materiales y recursos, etcétera), la modificación de los distintos currículums del centro y un impacto positivo en todos y todas las alumnas implicadas (como se verá al exponer los resultados al final del artículo).

\section{MÉTODO}

Para desarrollar nuestro trabajo investigador escogimos el modelo de investigaciónacción cooperativa, que nos pareció adecuado porque se relaciona con nuestro objetivo que consiste en: Iniciar una transformación del currículum del ciclo inicial de un centro de educación primaria para que avance hacia un modelo efectivo de educación intercultural utilizando como desencadenante un recurso musical. Cómo bien definen Bartolomé y Sandín (2001), este ocurre cuando se ven implicados el personal de dos o más instituciones; generalmente una de ellas orientada hacia la producción científica o a la formación de profesionales y la otra es una escuela o institución en la cual trabajan estos profesionales a los cuales se pretende formar. Ambas deciden agruparse (a demanda de unos u otras), para resolver juntos problemas que atañen a la práctica profesional de estos últimos, vinculando los procesos de investigación con los procesos de innovación y con el desarrollo y la formación profesional. Nuestra pretensión era que el profesorado implicado en el estudio entrase en un proceso autorreflexivo y transformador de su práctica educativa hacia la vertiente intercultural. El punto de partida de este cambio se produciría desde el trabajo de canciones en otras lenguas, empezando, a partir de aquí, un proceso de elaboración de recursos, adopción de estrategias, entre otros, que permitieran modificar el resto de las áreas de acuerdo con el modelo de educación intercultural, en su tercer nivel de concreción (trabajo en las aulas). Evidentemente, este proceso tendría una incidencia directa en el currículum, y la intención era que la experiencia tuviera también repercusión en los diferentes documentos del centro.

De acuerdo con Kemmis (1988: 197), el aterrizaje de este modelo investigador se ajustó a un proceso que se desarrolló en cuatro fases:

Cuadro 1. Fases de la investigación

\begin{tabular}{|l|l|}
\hline \multicolumn{1}{|c|}{ Fases } & \multicolumn{1}{|c|}{ Contenido } \\
\hline Reflexión inicial & $\begin{array}{l}\text { Sobre la situación a la luz de nuestra preocupación temática: fase de re- } \\
\text { conocimiento. Elaboración del marco teórico y preparación de materiales. }\end{array}$ \\
\hline Planificación & $\begin{array}{l}\text { Diseño del proceso, selección de la muestra, ajuste de los recursos y for- } \\
\text { mación inicial. }\end{array}$ \\
\hline Acción & Puesta en práctica del plan previsto y observación de su funcionamiento. \\
\hline Reflexión final & $\begin{array}{l}\text { Analizar indicadores, sintetizar, interpretar datos, validarlos, explicarlos y } \\
\text { extraer conclusiones. }\end{array}$ \\
\hline
\end{tabular}

Fuente: adaptado de Kemmis (1988: 197). 


\subsection{ESTRATEGIAS DE RECOGIDA DE LA INFORMACIÓN}

Hoy se acepta que no existe una única manera de conocer la realidad, explorarla, comprenderla y reconstruirla, y el interés por los hechos mensurables ha dado paso al interés por los significados e interpretaciones de los propios sujetos. Se aboga pues por la pluralidad de métodos y la adopción de diferentes estrategias que permitan captar la naturaleza abierta, dinámica y fluida de la realidad social y educativa (Bartolomé y Sandín, 2001; Mateo, 2001; Sandín, 2003). Desde esta perspectiva, se previeron utilizar las principales estrategias (en varias modalidades) de recogida de información dentro del modelo de investigación cualitativa: observación, análisis de documentos y materiales didácticos, entrevistas, hojas de anécdotas y diarios de sesiones. También se empleó un cuestionario en la primera fase del proceso investigador. En la siguiente tabla relacionamos las diferentes fases del proceso investigador con las técnicas de recogida de la información empleadas.

Cuadro 2. Estrategias de recogida de información según las fases de la búsqueda

\begin{tabular}{|c|c|c|c|c|c|c|c|}
\hline \multirow[b]{2}{*}{ FASES } & \multicolumn{2}{|c|}{ Estrategias } & \multirow[b]{2}{*}{ Cuestionario } & \multirow{2}{*}{$\begin{array}{c}\text { Análisis de } \\
\text { documentos } \\
\text { del centro y } \\
\text { materiales } \\
\text { didácticos }\end{array}$} & \multirow{2}{*}{$\begin{array}{l}\text { Observación } \\
\text { (notas de } \\
\text { campo y } \\
\text { hojas de } \\
\text { anécdotas) }\end{array}$} & \multirow{2}{*}{$\begin{array}{l}\text { Diarios } \\
\text { de } \\
\text { sesiones }\end{array}$} & \multirow[b]{2}{*}{ Entrevistas } \\
\hline & \multicolumn{2}{|c|}{$\begin{array}{l}\text { Fuentes de } \\
\text { información }\end{array}$} & & & & & \\
\hline \multirow{4}{*}{$\begin{array}{l}1^{\text {a }} \text { FASE: } \\
\text { Análisis de } \\
\text { contexto y } \\
\text { planificación } \\
\text { y diseño de la } \\
\text { intervención }\end{array}$} & \multicolumn{2}{|c|}{ Alumnado } & & $\sqrt{ }$ & & & \\
\hline & \multicolumn{2}{|c|}{ Profesorado } & $\sqrt{ }$ & & & & $\sqrt{ }$ \\
\hline & \multicolumn{2}{|l|}{ Centro } & & $\sqrt{ }$ & $\sqrt{ }$ & & \\
\hline & \multicolumn{2}{|c|}{ Reuniones de trabajo } & & & $\sqrt{ }$ & & \\
\hline \multirow{4}{*}{$\begin{array}{l}2^{\mathrm{a}} \text { FASE: } \\
\text { Formación y } \\
\text { transformación } \\
\text { curricular }\end{array}$} & \multirow{2}{*}{ AULAS } & Alumnado & & & $\sqrt{ }$ & & \\
\hline & & Profesorado & & & $\sqrt{ }$ & $\sqrt{ }$ & \\
\hline & \multicolumn{2}{|l|}{ Centro } & & & $\sqrt{ }$ & & \\
\hline & \multicolumn{2}{|c|}{ Reuniones de trabajo } & & & $\sqrt{ }$ & & \\
\hline \multirow{3}{*}{$\begin{array}{l}\mathbf{3}^{\mathbf{a}} \text { FASE } \\
\text { Incidencia y } \\
\text { permanencia } \\
\text { del cambio }\end{array}$} & \multicolumn{2}{|c|}{ Alumnado } & & $\sqrt{ }$ & & & \\
\hline & \multicolumn{2}{|c|}{ Profesorado } & & & & & $\sqrt{ }$ \\
\hline & \multicolumn{2}{|l|}{ Centro } & & $\sqrt{ }$ & & & \\
\hline
\end{tabular}

\subsection{ANÁLISIS DE LA INFORMACIÓN}

De acuerdo con las diferentes técnicas y estrategias de recogida de la información, utilizamos tanto programas informáticos de análisis, por un lado, para datos de tipo cuantitativo 
SPSS (Statiscal Package for Social Sciences), donde se introdujeron las diversas variables, se codificaron y se procedió a crear la matriz de respuestas mediante el vaciado de los cuestionarios. Tras el vaciado de datos y la creación de la matriz correspondiente, se realizó un análisis de tipo descriptivo, extrayendo tablas de frecuencias de cada una de las variables para permitir interpretar sus resultados. También se creyó conveniente utilizar las tablas de contingencia para cruzar varias variables entre ellas y permitir una mayor comprensión de los resultados.

Por otro lado, el análisis, interpretación y elaboración de los datos es un aspecto clave en la investigación cualitativa, en la que podemos hablar de un proceso cíclico inserto en todas las etapas de la investigación, y que tiene como objetivo contrastar, triangular, y validar toda la información obtenida para establecer conclusiones en referencia al objetivo último de la investigación. En este trabajo de investigación, para efectuar el análisis de los datos elaboramos dos sistemas de categorías establecidas previamente (a priori).

- Una primera tabla para recoger las dos dimensiones en cuanto al proceso de formación y transformación curricular donde cada dimensión se corresponde a una metacategoría de análisis.

- Una segunda tabla para analizar la valoración del profesorado participante y no participante en la experiencia una vez finalizada.

La información recogida por medio de las diferentes estrategias de recopilación de información (80 documentos) se transcribió íntegramente con un procesador de textos (Microsoft Word), y para el manejo y tratamiento de la información se utilizó el programa informático de análisis cualitativo ATLAS-Ti, tanto en la codificación de la información como en el proceso de organización y estructuración de los datos de acuerdo con el sistema categorial empleado en cada caso.

\section{RESULTADOS}

De acuerdo con nuestro objetivo general de conseguir iniciar una transformación curricular hacia la vertiente intercultural, asociada estrechamente a un proceso de formación del profesorado, creemos que se ha conseguido. Para corroborar esta afirmación, presentamos el mapa conceptual general correspondiente al proceso de la acción y donde destacan las 118 acotaciones correspondientes a actividades de innovación curricular intercultural en cuanto a integración de contenido y logro de objetivos curriculares. Estrechamente ligada aparecen las 78 acotaciones correspondientes a actividades de innovación metodológica en todos sus aspectos. Que el profesorado haya sido el artífice de estos logros demuestra, claramente, su propio proceso de formación. 
Figura 1. Mapa conceptual general

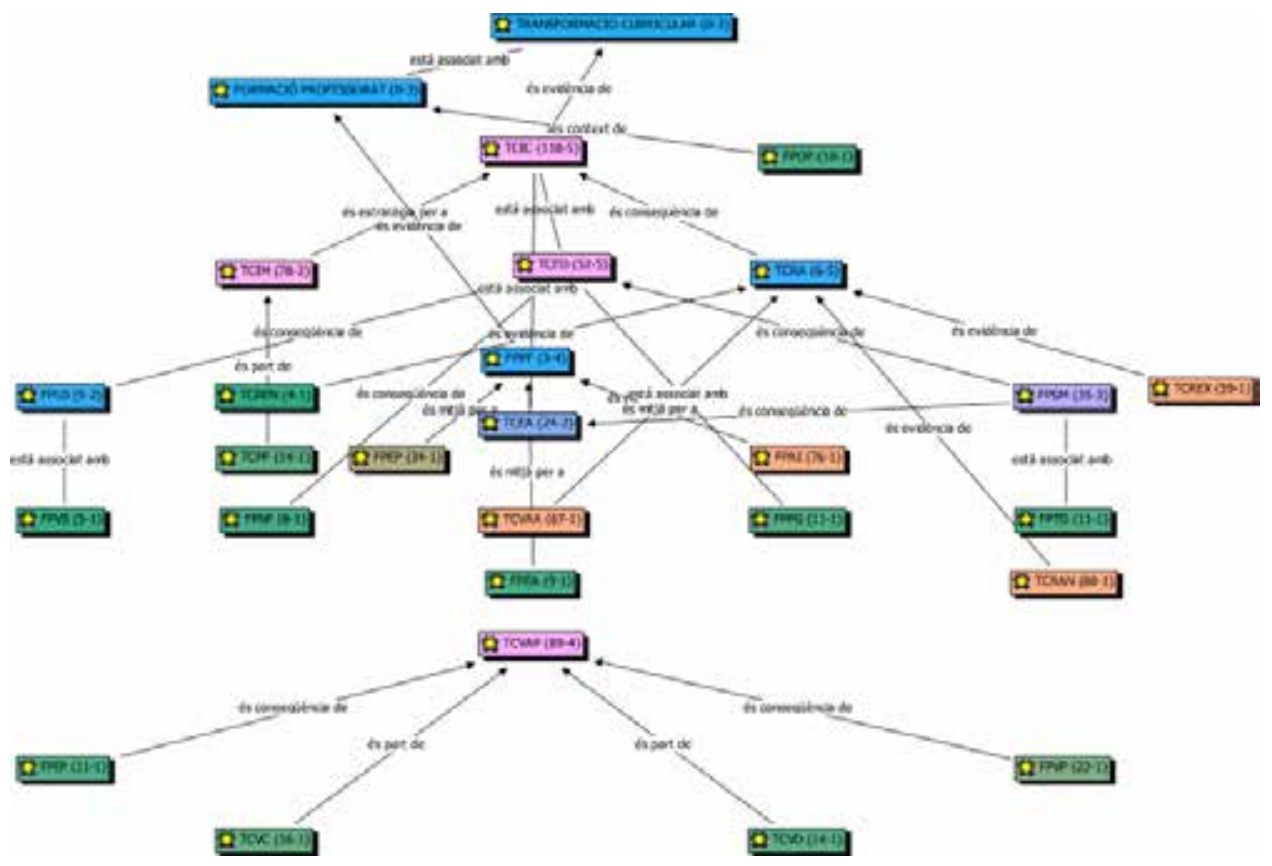

Destacamos que el objetivo general: "iniciar una transformación del currículum del ciclo inicial de un Centro de Educación Primaria para que avance hacia un modelo efectivo de educación intercultural utilizando como desencadenante un recurso musical", ha sido logrado con esta experiencia de investigación-acción cooperativa, lo que ha recompensado así el trabajo de todas las personas implicadas y ha demostrado que es posible adelantar en esta línea educativa, y que el profesorado devenga el motor del cambio educativo.

Además de este objetivo general, y contando que un verdadero proceso de transformación curricular debía afectar a los diferentes elementos de la comunidad educativa (Arnaiz, 2003; Gairín, 2004; Banks, 2006), agrupamos los objetivos específicos alrededor del profesorado, las estrategias metodológicas, el alumnado, el centro y el currículum.

\subsection{RESPETO AL PROFESORADO}

Se había planteado conseguir, por medio de la formación y el traspaso de información, que las características de un verdadero modelo de educación intercultural fueran conocidas por el máximo número de miembros del profesorado, de acuerdo con la idea del profesor como profesional reflexivo de Cabrera, Espín, Marín y Rodríguez (1999) y Díaz Aguado (1999), así como conocer las características del modelo de investigaciónacción como mejor manera de aunar teoría y práctica, de acuerdo con las indicaciones de Kemmis y Mctaggart (1988), Pérez Serrano (1998), Bartolomé y Sandín (2001), Sandín (2003) y Jordán (2004). 
La investigación-acción cooperativa fue vía de desarrollo profesional que propició la formación del profesorado y la transformación social. Efectivamente, ha sido la implicación del profesorado en todo el proceso investigador el medio por el que se ha logrado el conocimiento del modelo de Educación Intercultural y de cómo realizar un proceso de transformación curricular planteando objetivos de cariz intercultural en el currículum.

El proceso formativo ha venido dado por tres vías convergentes: desde el trabajo innovador de cada uno de los profesores participantes en la experiencia, desde las aportaciones del equipo investigador y desde la reflexión conjunta del grupo, esta constatación corrobora las ventajas del sistema cooperativo adoptado en la investigación.

El proceso de autoaprendizaje demostrado por los participantes, hasta consolidar diferentes vías de actuación y conseguir plena autonomía de trabajo, también es un indicador de las ventajas del sistema empleado. Se ha constatado un proceso de formación y de transformación de la práctica educativa de los prácticos desde la dependencia inicial de recursos y actividades facilitadas por la investigadora-formadora hasta lograr la total autonomía en la etapa final de la experiencia, demostrando una creatividad constante y una gran capacidad de innovación.

Entendemos, de acuerdo con las evidencias recogidas, que, efectivamente, se ha conseguido que el profesorado no solo conozca, sino que haya obtenido una confirmación de los beneficios del modelo de educación intercultural, y un interés que facilitará la implicación del resto de profesorado de la escuela en el trabajo con este modelo, de acuerdo con los efectos positivos que la experiencia ha tenido. El convencimiento que nos permite hacer estas afirmaciones, se encuentra en el hecho de recoger y analizar las valoraciones parciales y finales del profesorado participando respeto a su participación en la experiencia investigadora. Estas valoraciones positivas de los maestros están estrechamente vinculadas al impacto del trabajo realizado en su alumnado: por el interés y participación de los niños y niñas en general, por el protagonismo logrado por el alumnado nacido en otros países, por la mejora en la valoración del grupo hacia otros compañeros y compañeras, por la motivación de los grupos por las actividades realizadas, por el clima creado durante las sesiones de intercambio cultural y por el logro de objetivos curriculares desde un modelo de educación intercultural.

Planteamos una investigación-acción cooperativa con la intención de que el profesorado participante fuera haciendo suya la propia acción y se implicase, desde el principio, en una espiral de cambio. Esta espiral ha resultado ser antagónica según los protagonistas fueran el equipo de prácticos o la investigadora, ya que, efectivamente, tanto cuanto disminuía la participación de la investigadora externa, crecía el protagonismo y la participación del equipo de profesorado en su propio proceso de cambio.

La siguiente figura muestra gráficamente el desarrollo del proceso. 
Figura 2. Desarrollo de la acción

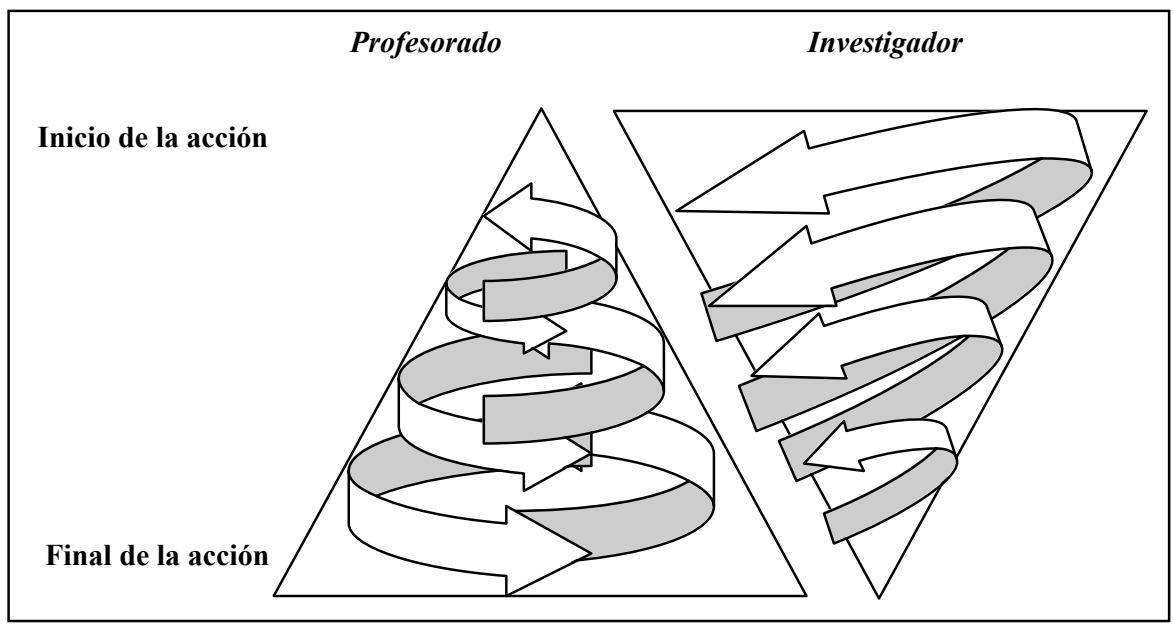

Lo más remarcable sería el trabajo en grupo que, gracias a la constante coordinación y buenas prácticas de los participantes en la experiencia, consiguieron superar las expectativas iniciales en todos los aspectos planteados.

\subsection{RESPETO A LAS ESTRATEGIAS METODOLÓGICAS}

De acuerdo con Besalú (2002) y Díaz Aguado (2003), entendíamos que no se produciría una verdadera transformación si el profesorado no conseguía adoptar también innovaciones didácticas que permitieran lograr mejor los objetivos propuestos: aprendizaje cooperativo, apoyo tecnológico, trabajo con vídeos, uso de Internet, creación de power-points, etcétera.

Pensamos que, en este aspecto, también el profesorado ha conseguido lograr un verdadero cambio en su práctica educativa, con una consolidación de diferentes vías metodológicas:

- Por la inclusión de las canciones en otras lenguas como eje de trabajo intercultural, en el caso de la especialista de música.

- Por la modificación de estrategias (grupos cooperativos), y recursos (búsqueda por internet), priorizando los objetivos curriculares, en el caso de la tutora de $2 \mathrm{C}$.

- Por la innovación en cuanto al planteamiento global de conocimiento cultural, participativo por el que se refiere a la estrategia de enseñanza-aprendizaje, y con apoyos de personas "expertas" como recurso más empleado, en el caso de la tutora de $2^{\text {a }}$.

- Por la búsqueda exhaustiva de materiales y la creación y presentación de montajes visuales, como eje de la transformación curricular, en el caso del tutor del grupo B.

Aunque cada uno de los profesores/as participantes ha adoptado diferentes metodologías de trabajo, entendemos que todas ellas han sido efectivas, y que, durante todo el proceso, el intercambio de ideas, materiales y recursos entre el profesorado, el equipo 
investigador, las familias, los "expertos culturales" y el propio alumnado ha sido una constante, lo que revaloriza el trabajo en grupo desde su perspectiva más amplia.

Se planteó también como innovación metodológica la implicación de las familias en actividades escolares, propiciando su participación en el proceso de transformación curricular previsto, tal y como recogíamos de las recomendaciones de Arnaiz (2003) y Gairín (2004). Pensamos que, efectivamente, se ha conseguido abrir la escuela a las familias, dándoles una oportunidad de colaborar y participar activamente en la escuela, que en todos los casos ha superado ampliamente las expectativas que podíamos tener al respeto.

\subsection{RESPETO AL CENTRO}

Queríamos que la escuela se hiciera eco de la experiencia y los diferentes documentos del centro, (en el segundo nivel de concreción curricular) se vieran modificados en este sentido, así como expandir e interesar en el proyecto a todo el claustro escolar, buscando la consolidación del cambio (Bartolomé y Sandín, 2001).

Se ha conseguido que el centro adopte el modelo de educación intercultural propuesto e incorpore el trabajo realizado al trabajo del Centro mediante un Plan de innovación docente aprobado por el claustro y presentado al Departamento de Educación como proyecto de la escuela. Esta constatación acontece el mejor indicador de la permanencia y cristalización del cambio que planteábamos.

La toma de conciencia de los beneficios de adoptar un modelo educativo intercultural, desde la propia práctica educativa de los participantes, entendemos que será crucial a la hora de expandir e interesar al resto del profesorado en la transformación curricular deseada para toda la escuela.

\subsection{RESPETO AL CURRÍCULUM}

Evidentemente el currículum, en su tercer nivel de concreción, debía introducir nuevos objetivos y contenidos que dieran conocimiento de otras culturas, inicialmente mediante una canción de los diferentes países representados en las clases y profundizando posteriormente en las otras materias Besalú (2002). Podemos afirmar que se ha conseguido por medio de:

- La obtención de materiales y recursos culturales nuevos.

- Pautas de desarrollo curricular para la creación o modificación de unidades de programación para todas las áreas.

Destacamos por un lado, el hecho de elaborar y validar materiales y recursos nuevos: el cancionero, los montajes visuales, los mapas, las plantillas y dibujos específicos, los murales y los listados de palabras en otras lenguas.

Por otro lado, la utilización y validación de materiales (de cariz intercultural o, simplemente, de información cultural) ya existentes, ha sido también una novedad muy provechosa: uso de vídeos promocionales de diferentes países, trabajo con cuentos populares, juegos diversos, colecciones específicas ("Yo soy de...", "Yo vengo de...", "Un paseo por...") y la búsqueda de información cultural mediante internet. 
Evidentemente, la validación de estos nuevos recursos y materiales ha sido posible porque se han creado o modificado las unidades de programación para todas las áreas, teniendo como muestra las unidades iniciales para trabajar el conocimiento de Brasil y las sugerencias de trabajo por áreas. Por la complejidad y densidad del trabajo realizado, creemos que se pueden tomar como pautas de desarrollo de cualquier ulterior acción en este sentido.

\subsection{RESPETO AL ALUMNADO EN GENERAL}

Se quería promover la competencia del alumnado en múltiples culturas, reconociendo las semblanzas entre ellas, promoviendo el diálogo cultural desde la igualdad y apreciando la diversidad étnica y cultural como elemento positivo, de acuerdo con las indicaciones del modelo educativo intercultural de Marín (2001), Sabariego (2002), Jordán (2004), Aguado, Gil y Mata (2005) y Banks (2006). Creemos que respeto al alumnado en general se ha conseguido:

- Un mejor desarrollo de las competencias interculturales, logrado con la máxima naturalidad.

Esta afirmación se basa en la constatación que el alumnado ha mostrado, sin lugar a dudas, un gran interés por conocer otras culturas.

La participación e implicación del alumnado en todas las actividades propuestas también es un claro indicador de este logro de competencias interculturales.

Haber conseguido que la valoración de los niños y niñas nacidos en otros países haya aumentado tras la experiencia acontece otro de los efectos que nos permiten afirmar que se ha adelantado hacia un modelo de educación intercultural.

Las reacciones emocionales de satisfacción recogidas a lo largo del proceso resultan también un indicador de la bondad de la experiencia y de la naturalidad con la que ha sido recibida.

Finalmente hemos visto en numerosas ocasiones que el aprendizaje ha sido significativo, indicador que corrobora la efectividad de la transformación curricular pretensa.

\subsection{RESPETO AL ALUMNADO EXTRANJERO}

Uno de los principales objetivos específicos de la investigación se centraba en estimular la autoestima del alumnado nacido en otros países, ayudando así a la creación de identidades culturales personales, fomentando a la vez su participación. Todos los indicadores recogidos nos permiten afirmar que, efectivamente, se ha conseguido:

- Un "protagonismo" dirigido que nos ha permitido estimular su autoestima ayudándolos a formar su propia identidad personal.

Los elementos analizados y valorados que demuestran esta afirmación tienen que ver con la constatación de que el alumnado nacido en otros países ha demostrado una mejora de la participación dentro del grupo-clase. Su constante implicación en el desarrollo de las actividades es el indicador más palpable que el proceso investigador 
ha tenido efectos directos sobre el alumnado extranjero, con un notable incremento de la satisfacción personal y, consecuentemente, una mejora de la autoestima al sentirse protagonista.

\section{DISCUSIÓN}

Evidentemente, como comentario general, creemos que los resultados en todos los ámbitos son altamente satisfactorios, puesto que al plantear un proyecto con incidencia directa en las aulas, en un proceso continuo de formación permanente del profesorado, y con recursos específicos y constantemente reflexionados, mejorados y aplicados, los beneficios y beneficiarios han sido diversos.

Finalmente, constatar que nuestra hipótesis inicial de dar a conocer elementos culturales diversos al alumnado, propiciando el diálogo cultural desde la igualdad para conseguir un reconocimiento y un aprecio de los mismos, parece haberse confirmado ampliamente con la experiencia investigadora.

Más allá de la experiencia, y contando con que la escuela acabará incorporando estos materiales y este cambio educativo a todo el centro (como el plan de innovación docente aprobado por el claustro nos indica), creemos importante destacar que se han creado una serie de recursos específicos de cariz intercultural que han sido probados y validados por la investigación, lo que cubre algo el vacío respecto de la falta de recursos específicos y que podría extenderse a otros centros donde, con unas sesiones de asesoramiento hechas por personal cualificado, ayudaría a dar respuesta a esta necesidad de conseguir adoptar un modelo educativo verdaderamente intercultural.

Por último, también destacamos los beneficios para los miembros del equipo de investigación y de otros profesionales dedicados a la investigación educativa, que verán una investigación donde la complementariedad metodológica permite abordar una temática actual y compleja desde perspectivas diferentes.

\section{REFERENCIAS BIBLIOGRÁFICAS}

Aguado, M., Gil, I. y Mata, P. (2005). Educación intercultural: Una propuesta para la transformación de la escuela. Madrid: Los Libros de la Catarata.

Aja, E. (2000). La regulación de la educación de los inmigrantes. En VV.AA, La inmigración extranjera en España. Los retos educativos (pp. 69-98). Barcelona: Fundació La Caixa.

Alegre, M. A. (2005a). Educació i immigració: L'acollida als centres educatius. Barcelona: Fundació Jaume Bofill.

(2005b). Geografies adolescents a secundària. Els posicionaments dels fills i filles de famílies d'origen immigrat en els mapes relacionals $i$ culturals articulats en l'àmbit escolar. Tesis doctoral. Barcelona: Departament de Sociologia, Universitat Autònoma de Barcelona. Recuperat el 25 de novembre de 2012 des de http://www.tdx.cat/TDX-0714105-162736

Aranda, V. (2011). Reflexión y análisis de políticas y prácticas innovadoras a la luz de las representaciones sociales y de la necesidad de una educación intercultural en la formación inicial docente. Estudios Pedagógicos, vol. 37 (2), 301-314.

Arnaiz, P. (2003). Educación inclusiva: Una escuela para todos. Málaga: Aljibe.

Atwater, M. (2010). Multicultural science education and curriculum materials. Science Activities, vol. 47 (4), 103-108. 
Banks, J. (2006). Race, culture and education: The selected works of James A. Banks. New York: Routledge.

Bartolomé, M. (Coord.). (2002). Identidad y ciudadanía. Un reto a la educación intercultural. Madrid: Narcea.

\section{Barcelona.}

. y Sandín, M. (2001). Metodologia qualitativa en Educació. Barcelona: Universitat de (1997). Diagnóstico a la escuela multicultural. Barcelona: Cedecs.

Bennett, M. J. (2009). Defining, measuring, and facilitating intercultural learning: A conceptual introduction to the intercultural education double supplement. Intercultural Education, vol. 20 (4), 1-13.

Besalú, X. (2002). Diversidad cultural y educación. Madrid: Síntesis.

Cabrera, F., Espín, J., Marín, M. y Rodríguez, M. (1999). La formación del profesorado en educación multicultural. En M. A. Essomba (Coord.), Construir la escuela intercultural. Reflexiones y propuestas para trabajar la diversidad étnica y cultural (pp. 75-80). Barcelona: Graò.

Delgado, M. (1998). Diversitat i integració. Barcelona: Empúries.

De Lucas, J. (2003). La inmigración, como res política. En J. Chamizo de la Rubia y K. Yamagne (Coords.), Movimientos de personas e ideas y multiculturalidad (Vol. II) (pp. 193-225). Bilbao: Universidad de Deusto.

Díaz Aguado, M. (2003). Educación intercultural y aprendizaje cooperativo. Madrid: Pirámide. (1999). Aprendizaje cooperativo y educación intercultural. Investigación-acción en centros de primaria. Psicología Educativa, vol. 5 (2), 141-200.

Eresta, M. y Delpino, M. (2007). La inserción de los adolescentes latinoamericanos en España: Algunas claves. Madrid: Observatorio de la infancia, Ministerio de trabajo y asuntos sociales.

Etxeberría, F. y Elosegui, K. (2010). Integración del alumnado inmigrante. Revista Española de Educación Comparada, (16), 235-263.

Eurydice. (2009). La integración escolar del alumnado inmigrante en Europa. Bruselas: Unidad Europea de Eurydice.

European Unit.

(2004). Integrating immigrant children into schools in Europe. Brussels: Eurydice

Gairín, J. (2004). Organizar la escuela intercultural: Una exigencia de futuro. XIII Congreso Nacional y II Iberoamericano de Pedagogía. La educación en contextos multiculturales: Diversidad e identidad (pp. 282-303). Valencia: Sociedad Española de Pedagogía.

Gelpi, E. (1998). Interculturalidad, dominaciones y conflictos internacionales. En X. Besalú, G. Campani y J. M. Palaudàrias (Comps.), La educación intercultural en Europa. Un enfoque curricular (pp. 21-35). Barcelona: Pomares-Corredor.

Goytisolo, J. y Naïr, S. (2000). El peaje de la vida: Integración o rechazo de la emigración en España. Madrid: Aguilar.

Jordán, J. (2007a). Educar en la convivencia en contextos multiculturales. En E. Soriano (Coord.), Educación para la convivencia intercultural (pp. 59-98). Madrid: La Muralla. (12), 59-80.

(2007b). Formación intercultural del profesorado de secundaria. Estudios sobre Educación,

(2004). La formación permanente del profesorado en educación intercultural. En J. Jordán, X. Besalú, M. Bartolomé, M. Aguado, C. Moreno y M. Sanz (Coords.), La formación del profesorado en educación intercultural (pp. 11-48). Madrid: Los Libros de la Catarata. . (1996). Propuestas de educación intercultural para profesores. Barcelona: CEAC.

Kemmis, S. (1988). El currículum: Más allá de la teoría de la reproducción. Madrid: Morata. y Mctaggart, R. (1988). Cómo planificar la investigación-acción. Barcelona: Laertes.

Marín, M. (2001). El tratamiento de la interculturalidad en la educación primaria. En T. Pozo, R. López, B. García y E. Olmedo (Coords.) Investigación educativa: Diversidad y escuela (pp. 224-257). Granada: Grupo Editorial Universitario. 
Mateo, J. (2001). Investigació educativa (Disc compacte). Barcelona: Universitat de Barcelona.

Mussino, E. \& Strozza, S. (2012). The delayed school progress of the children of immigrants in lower secondary education in Italy. Journal of Ethnic and Migration Studies, vol. 38 (1), 41-57.

Naïr, S. (2001). La inmigración explicada a mi hija. Barcelona: Plaza y Janés.

Navarro, J. (Coord.). (1994). Anàlisi crítica del marc curricular de la reforma des d'una perspectiva intercultural. Barcelona: Universitat de Barcelona.

Palou, B. (2010). La integración de la juventud de origen magrebí en Cataluña. Tesis doctoral. Barcelona: Departament de Mètodes i Diagnòstic en Educació, Universitat de Barcelona. Recuperat el 25 de novembre de 2012 des de http://tdx.cat/handle/10803/2363

Pavez, I. (2011). Migración infantil: Rupturas generacionales y de género. Las niñas peruanas en Barcelona y Santiago de Chile. Tesis doctoral. Barcelona: Departament de Sociologia, Universitat Autònoma de Barcelona. Recuperado el 25 de noviembre de 2012 desde http://www. tdx.cat/handle/10803/79139

Pérez, C. y Rahona, M. (2005). La integración de los inmigrantes en el sistema educativo. Recuperado el 14 de noviembre de 2013 desde http://www.unavarra.es/migraciones/papers3/ Comunicacion\%2011Perez-Rahona.pdf

Pérez Serrano, G. (1998). Investigación cualitativa. Retos e Interrogantes II. Madrid: La Muralla.

Perry, L. \& Southwell, L. (2011). Developing intercultural understanding and skills: Models and approaches. Intercultural Education, vol. 22 (6), 453-466.

Reid, C. \& Sripraks, A. (2012). The possibility of cosmopolitan learning: Reflecting on future directions for diversity teacher education in Australia. Journal of Teacher Education, vol. 40 (1), 15-29.

Rubio, M. (2009). El desarrollo de la competencia comunicativa intercultural en la formación inicial docente. Estudios Pedagógicos, vol. 35 (1), 273-286.

Sabariego, M. (2002). La educación intercultural ante los retos del siglo XXI. Bilbao: Desclée De Brouwer.

Sadker, M., Sadker, D. \& Zittleman, K. (2008). Teachers, schools, and society. New York: McGraw-Hill.

Sandín, M. (2003). Investigación cualitativa en educación: Fundamentos y tradiciones. Madrid: McGraw-Hill.

Siguán, M. (1998). La escuela y los inmigrantes. Barcelona: Paidós.

Stickel, G. W. (1987). Cultural pluralism and the schools: Theoretical implications for the promotion of cultural pluralism. Annual Conference of the American Association of Colleges for Teacher Education. Washington, D.C. United States.

Talkington, B., Lengel, L. \& Byram, M. (2004). Setting the context, highlighting the importance: Reflections on interculturality and pedagogy. Retrieved September 3, 2013 from www.llas. ac.uk/resources/paper.aspx? resourceid=2048

Terrén, E. (2003). Educación democrática y ciudadanía multicultural: El reaprendizaje de la convivencia. Revista Praxis, (3), 5-28.

Tosolini, A., Giusti, S. e Papponi Morelli, G. (2007). A scuola di intercultura. Cittadinanza, partecipazione, interazione: Le risorse della società multiculturale. Gardolo: Centro Studi Erickson. 
